\title{
Analysis of Cloud Workflow System
}

\author{
Hailan $\mathrm{Pan}^{*}$ and Cuihong Wu \\ School of Economics and Management, Shanghai Polytechnic University, 360Hao, JinHaiLu, PuDong Shanghai 201209 China \\ ${ }^{*}$ Corresponding author
}

\begin{abstract}
With the rapid development of economy, science and technology, the computing environment and process become more and more complicated, and the computing scale becomes larger and bigger, The cloud workflow system can solve the automatic control and comprehensive management of transaction flow in big data environment. This paper introduces the current situation of cloud workflow technology at home and abroad, and then analyzes its related technologies, including model, scheduling method and Hadoop technology. At last, the paper elaborates the MapReduce workflow system using the middle layer Proxy structure and explains its advantages.
\end{abstract}

Keywords-component; HPC; workflow; Petri Net; Hadoop; MapReduce

\section{INTRODUCTION}

With the rapid development of Internet technology, Many fields have produced a lot of data, from business data, which come from the network interactive media data, from GPS devices, from FRID equipment and a variety of monitoring equipment, sensor data. According to the statistics the global appears 2.5EB data each month, so how to collect and use data to carry out cooperation of different industries in different areas is the focus of the current data studies[1]. Cloud computing is the first choice for data storage and data processing, and the strength of workflow system is to solve collaborative data processing of the interdisciplinary and interdisciplinary. Therefore, this paper studies the cloud workflow scheduling algorithm, and explores the advantages which MapReduce scheduling model handles centralized central engine bottlenecks.

\section{RESEARCH StATUS LOCAL AND ABROAD}

Workflow technology which has emerged from the 1980s, has gradually become the core of all types of information systems, especially in the last decade, workflow technology has experienced rapid development because especially HPC(highperformance computing) widely used that makes enterprise business processes Processing more and more complex. In order to solve the collection, storage, access, calculation and management of a series of issues, the concept of cloud workflow or workflow management system running in parallel environment brings out. Researchers build a framework for MapReduce to work with workflows and take advantage of their respective strengths.

At present, the workflow management system running on the parallel environment is GridFlow, Kepler, Taverna, Pegasus, Oozie and so on. Among them, Kepler is a workflow management Java-based, which developed on the basis of
Ptolemy II and inherited its Inherited the characteristics of its role-oriented modeling; Triana supports multiple languages including WSFL(Web Service Flow Language), BPEL(Business Process Execution Language), DAG(Directed Acyclic Graph) and Perinet formats; Pegasus uses the proprietary DAX language which is an XML representation of the abstraction layer based on DAG language; Oozie is an open-source workflow engine which is produced for Apache Hadoop by Yahoo, that is used to manage and coordinate Jobs running on the Hadoop platform (including HDFS, Pig and MapReduce)[2-6] .

These systems have promoted the research and application of workflow, and provided strong support for the efficiency improvement and standardization management of enterprises and scientific research organizations. However, how to further unify the system specification, excavate the potential of the system and improve the efficiency of the system , shorten the total execution time and re-development cycle of the business process is still an important issue that has attracted much attention in academia and industry [7].

\section{DESCRIPTION OF THE RELATED TECHNOLOGY}

\section{A. Workflow Model}

Workflow model is an abstract representation of the whole workflow, as well as an abstract representation of the whole operation and management process. Workflow modeling is one of the most important parts of workflow management system. Different workflow models, corresponding workflow representations and descriptions, engine design and implementation will be different. According to the different direction of the workflow management system, the workflow can be divided into the control flow model, the data flow model, and the mixed model. SWF(The scientific workflow) is mainly oriented to data flow, and focuses on the data dependency among tasks, so SWF can adopt the model of data flow.

At present, there are a variety of formal techniques to represent the workflow process, the common workflow logic process has Petri nets, directed graphs and UML.

\section{1) Petri net}

Petri net is a graphical mathematical representation of discrete systems. First of all it has a precise definition to avoid the ambiguity, uncertainty and contradiction; Secondly, the formal system of rigorous process can be used to reflect on the process and promote usage of a lot of analytical techniques. Petri nets have a more extensive description and it can express the order, concurrency, loops, condition triggering and other 
events, and the state of the task in the workflow, shown in Figure 1.

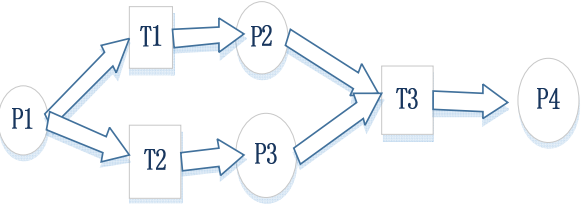

FIGURE I. SIMPLE PETRI NET

\section{2) Directed acyclic graph}

DAG is Directed acyclic graphs, in Figure 2 a node is represented a task (or an activity), the arc is represented the previous dependencies among with the task, and sometimes the arc to set the weight is represented the property or the required parameters. DAG can express the order, parallel, and conditions such as control structures, but does not support loop structure. DAG is simple and intuitive features, but its expression is relatively limited.

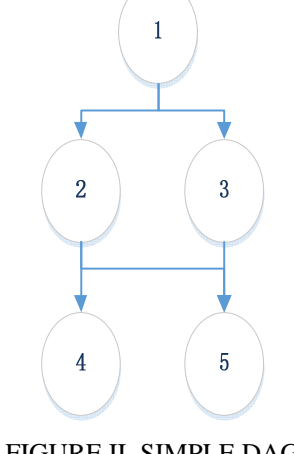

\section{3) $U M L$}

UML is an modeling language which is object-oriented, modeling and graphical which uses a unified standard definition and labeling to achieve the description and modeling of the software. UML is a common standard modeling language, which is not only for software development, part of the map can also be used for enterprise modeling, business engineering, system configuration and process analysis.

\section{B. Workflow Scheduling Method}

Task scheduling is the core of the workflow management system. Workflow task scheduling, including the decomposition of the task, resource positioning, resource selection and optimization. At present, the main scheduling algorithms are divided into three categories, including heuristic scheduling algorithm, agent-based task scheduling and economic grid scheduling algorithm, in which heuristic scheduling algorithm accesses favors from many researchers because of its computational overhead is small.

The heuristic algorithm can find the best solution within the acceptable computational cost, but it can not guarantee the optimality and feasibility of the solution, even in most cases, it can not explain the approximation between the optimal solution and the obtained solution. Heuristic scheduling algorithms are including GA(genetic algorithm), PSO(particle swarm algorithm), ant ACO(colony algorithm), ANN(neural network algorithm,) Sufferage algorithm, and HEFT(Hetero-geneous Earliest-Finish-Time) algorithm, etc.

GA can solve some large and complex scheduling problems, and that is proved the efficiency of the algorithm in dynamic distributed environment; PSO is a random search algorithm, which uses group intelligence model, and then establish the problem gradually in the search space optimization solution; ACO is an optimization algorithm that simulates the foraging behavior of ants, and which has the characteristics of concurrency and scalability. ANN is in the agent-based management model. Each agent uses a neural network scheduling algorithm to schedule and manage tasks, and uses an annealing algorithm to avoid the scheduling algorithm into a local optimal solution. Sufferage algorithm is to compare the last task which has been assigned to a computing node with the tolerance value of the current task to be assigned to, and the tasks which have bigger tolerance value are priority assigned; HEFT algorithm is belong to a list scheduling algorithm in heuristic algorithm. It consists of two phases: the task priority ranking stage, which calculates the value of the upward rank by the average computation amount and the average traffic volume, and calculates the priority order of the task queue by the value; processor selection phase, which according to the queue processes the task in order, and assigns (or inserts) the task into the processor which can provide the smallest earliest completion time.

\section{Hadoop}

Hadoop was created by Doug Cutting, founder of Apache Lucene. Hadoop mainly consists of two parts including the bottom of the HDFS (Hadoop Distributed File System) and the MapReduce engine on the upper level. HDFS is responsible for storing all the files of the storage node in the Hadoop cluster; The MapReduce engine mainly includes Job Trackers and Task Trackers. HDFS is designed to be deployed in a low-cost hardware cluster, and with high fault tolerance to ensure data reliability. Hadoop will be divided into a number of small tasks to the task (task) to perform, these small tasks are divided into two categories including map tasks and reduce tasks. Users only need to customize the map and reduce functions and don't worry about complex processing details.

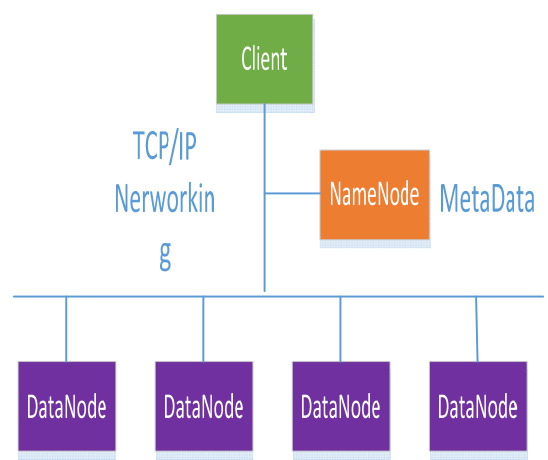

Replicated data blocks

FIGURE III. A SIMPLIFIED VIEW OF A HADOOP CLUSTER 


\section{MAPREDUCE WORKFLOW SySTEM}

\section{A. Introduce}

Workflow Framework based on MapReduce which is a workflow system for cloud computing is the focus of the following work, although this framework is relatively simple, but because MapReduce is a classic low-level framework in the cloud environment, the MapReduce workflow framework is a microcosm of the cloud framework, the research results will also has a corresponding significance for further comprehensive study of cloud framework .

We use the centralization architecture of service orchestrations, and we are also to improve it, penetrate the essence of distributed. In the system by adding Proxy layer to support the control by the central engine, the data between agents can be translated directly, and the system can avoid the bottleneck as far as possible. And for the cloud-based part of MapReduce, it can be assigned to the Hadoop model in the underlying cloud, so that it can fully play the advantages of distributed management.

\section{B. Workflow System}

Figure 4 depicts the overall architecture of the cloud workflow deployed in the cloud computing environment, including the user access layer, the workflow engine layer, the newly embedded intermediate agent layer, and the underlying cloud.

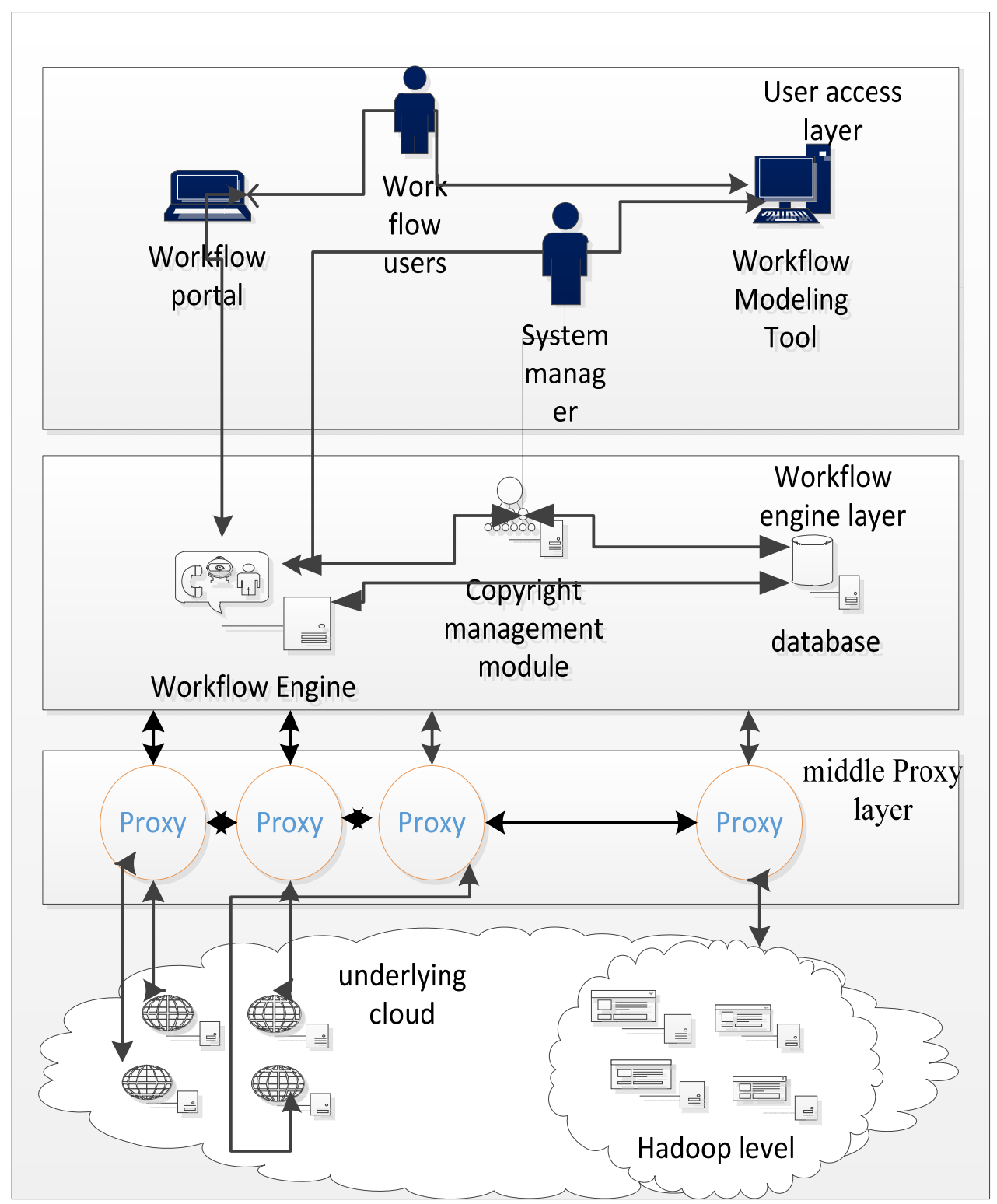

FIGURE IV. CLOUD WORKFLOW SYSTEM ARCHITECTURE 


\section{1) User access layer}

As a workflow portal user portal is information exchange channels between the user and the workflow engine, which can be installed client or web page. On the one hand, it displays the corresponding services provided in the system to the user, and provides the process service related information, and feedbacks the results of the submitted task execution. On the other hand, the user can select the required task flow in his available task list, and who can also customize the interface from the process, through the graphical modeling tools to carry out special process definitions, and provide real-time monitoring for implementing the process and so on. Of course, different users will have different permissions, so the services will also be different. In this paper, the DAG visualization interface is selected to model, because simple and intuitive DAG model are provided to facilitate the subsequent scheduling strategy research.

\section{2) Workflow engine layer}

With the development of information technology and the explosion of data collection speed, in addition to scientific research, the commercial nature of the workflow also increasingly tends to be computationally intensive, so the integrated workflow engine in the cloud environment has become a research hots which has high performance, high stability and good scalability.

\section{3) Supporting the underlying cloud}

Supporting the underlying cloud is based on cloud resources which is the bottom of the entire system, including transaction processing, storage and some other cloud support functions. As a distributed environment with high performance, low cost and high stability, the cloud computing environment is increasingly accepted by all circles. It is the main carrier of massive data processing and the main trend of future development.

\section{4) The middle layer proxy structure}

The service model, the control flow and the data flow have to pass through the central engine, so the data of the central engine are generally larger. On this basis, the distributed idea is added in this paper, Proxy nodes are used to manage the data nodes , While the agent is controlled by the central engine, which will allow only the control flow through the central engine, large data flow between the agents in a point to point transmission, the total transmission of data is about half the original, and transmission data is less after the central engine, but only the corresponding control information[8].

\section{The Advantage}

This intermediate agent architecture will bring significant advantages to the system, and the advantages of the intermediate broker architecture are the following:

\section{1) Lightweight and simple to deploy}

Proxy installation does not need to write any additional code, and can be remotely and dynamically configure its parameters, It only needs to track the services that the WSDL describes which goes through the proxy.

\section{2) Interference is small}

The middle-tier proxy architecture is independent. It can be deployed without interfering with existing services. Workflows use it with little change. This flexibility allows a gradual change infrastructure to handle large amounts of data when you can focus on improving data transfer between services.

The agent of the system is a circulation architecture, which makes it easier for the system to detect and handle failures. Agents themselves are connected through the WSDL interface, which allows them to be embedded into workflows or other higher-level Web services. Like other modules connected through the WSDL interface, the combined services can be managed through a workflow management system.

\section{CONCLUSION}

This paper mainly focuses on the workflows running on cloud computing platform. In order to solve the bottleneck problem when dealing with large data under the framework of traditional workflow, this paper proposes to add the middle layer Proxy (Agent) structure to the workflow framework . The engine manages the resource nodes through the control flow with the proxy, so that the data flow can be transmitted point to point between the nodes without having to pass through the central engine, thus greatly reducing the bottleneck. Because of the classical framework of MapReduce and Hadoop, we will study the MapReduce workflow scheduling model as a microcosm of cloud workflow, and analyze its scheduling model and scheduling environment.

\section{ACKNOWLEDGMENT}

This paper is sponsored by management science and engineering project of shanghai polytechnic university (No. XXKPY1606)

\section{REFERENCES}

[1] LiJun.Big Data Practice[M],2015, Beijing:Tsinghua University Press.

[2] http://gridflow.ca/[Z].

[3] http://kepler-project.org/[Z].

[4] http://www.taverna.org.uk/[Z].

[5] http://pegasus.isi.edu/[Z].

[6] http://oozie.apache.org/[Z].

[7] Altintas I, Barney O, Jaeger-Frank E. Provenance collection support in the kepler scientific workflow system. Springer Berlin Heidelberg, pp. 118-132,2006.

[8] Chen W, Wu J, Jiao L. State - feedback stabilization for a class of stochastictime - delay nonlinear systems. International Journal of Robust and Nonlinear Control, vol. 22 No 17, pp. 1921-1937, 2012. 